\title{
The urologic epithelial stem cell database (UESC) - a web tool for cell type-specific gene expression and immunohistochemistry images of the prostate and bladder
}

\author{
Laura E Pascal*1,2, Eric W Deutsch², David S Campbell2, Martin Korb², \\ Lawrence D True ${ }^{3}$ and Alvin Y Liu ${ }^{1,2}$
}

\begin{abstract}
Address: ${ }^{1}$ Department of Urology, and the Institute for Stem Cell and Regenerative Medicine, University of Washington, Seattle WA 98195, USA, 2Institute for Systems Biology, Seattle WA 98103, USA and ${ }^{3}$ Department of Pathology, University of Washington, Seattle WA 98195, USA

Email: Laura E Pascal* - lpascal@systemsbiology.org; Eric W Deutsch - edeutsch@systemsbiology.org;

David S Campbell - dcampbell@systemsbiology.org; Martin Korb - mkorb@systemsbiology.org; Lawrence D True - ltrue@u.washington.edu; Alvin Y Liu - aliu@u.washington.edu

* Corresponding author
\end{abstract}

Published: II December 2007

BMC Urology 2007, 7:19 doi:10.1186/1471-2490-7-19
Received: 22 June 2007

Accepted: II December 2007

This article is available from: http://www.biomedcentral.com/I47/-2490/7//9

(C) 2007 Pascal et al; licensee BioMed Central Ltd.

This is an Open Access article distributed under the terms of the Creative Commons Attribution License (http://creativecommons.org/licenses/by/2.0), which permits unrestricted use, distribution, and reproduction in any medium, provided the original work is properly cited.

\begin{abstract}
Background: Public databases are crucial for analysis of high-dimensional gene and protein expression data. The Urologic Epithelial Stem Cells (UESC) database http:// scgap.systemsbiology.net/ is a public database that contains gene and protein information for the major cell types of the prostate, prostate cancer cell lines, and a cancer cell type isolated from a primary tumor. Similarly, such information is available for urinary bladder cell types.

Description: Two major data types were archived in the database, protein abundance localization data from immunohistochemistry images, and transcript abundance data principally from DNA microarray analysis. Data results were organized in modules that were made to operate independently but built upon a core functionality. Gene array data and immunostaining images for human and mouse prostate and bladder were made available for interrogation. Data analysis capabilities include: (I) CD (cluster designation) cell surface protein data. For each cluster designation molecule, a data summary allows easy retrieval of images (at multiple magnifications). (2) Microarray data. Single gene or batch search can be initiated with Affymetrix Probeset ID, Gene Name, or Accession Number together with options of coalescing probesets and/or replicates.

Conclusion: Databases are invaluable for biomedical research, and their utility depends on data quality and user friendliness. UESC provides for database queries and tools to examine cell typespecific gene expression (normal vs. cancer), whereas most other databases contain only whole tissue expression datasets. The UESC database provides a valuable tool in the analysis of differential gene expression in prostate cancer genes in cancer progression.
\end{abstract}

\section{Background}

Public databases for the storage and retrieval of genomic and proteomic data have become an integral component of biomedical research. These databases can aid in the identification of genes and proteins responsible for disease and health and defining their function by enabling 
investigators in diverse research areas and interests with a range of computer expertise to have ready access to the stored information through one user interface. Previously, the Prostate Expression Database (PEDB) established a centralized archive of gene expression information for human prostate [1]. This database contains a large cDNA library of gene sequences obtained for normal/benign, benign prostatic hyperplasia (BPH), prostatic intraepithelial neoplasia (PIN) and malignant prostate disease states. The Prostate Gene Database (PGDB) is another prostate database that stores factual data about genes related to the human prostate and prostatic diseases supported by literature references [2]. These genes are grouped under molecular events of amplification, mutation, gross deletion, methylation, polymorphism, overexpression and linkage. These two databases provide valuable information obtained from whole prostate tissue. The characterization of tissues based on cell-surface protein expression [3] allows the possibility of separating cells of interest from that tissue for gene array analysis and determination of cell-type specific transcriptomes [4]. Public availability of cell-type specific data will be an important additional tool in future studies.

The Stem Cell Genome Anatomy Project (SCGAP) initiated by the National Institute of Diabetes and Digestive and Kidney Diseases (NIDDK) included seven organ-specific groups that were funded to form a research consortium. The aims of this consortium were to collectively develop necessary biological procedures and reagents for characterization of tissue specific progenitor cells and to characterize gene expression patterns in these cells using advanced technologies and bioinformatic techniques. The official web portal for SCGAP [5] was designed to deliver an overview of the progress of the consortium's research efforts and to function as a gateway to the websites of the consortium participants. As such, the detailed data, protocols and descriptions are accessible from the respective website of the participating SCGAP projects.

Our group, the urologic epithelial stem cells project, investigated the molecular basis of the differentiation of epithelial cells of the human prostate and bladder. We are interested in studying development and the cancer process in the context of interaction between individual cell types. Expression levels of CD cell surface antigens was first used to distinguish the constituent cell types of the prostate, as well as cancer cells from their normal counterpart $[3,6]$. The cell $\mathrm{CD}$ phenotyping data acquisition involved immunohistochemistry with 200 commercially available CD monoclonal antibodies (BD-PharMingen). Magnetic cell sorting (MACS) based on the cell typespecific CD expression was then used to isolate the following prostatic cell types: CD31+ endothelial cells, CD26+ luminal secretory and CD104+ basal cells of the epithe- lium, and $\mathrm{CD} 49 \mathrm{a}^{+}$fibromuscular cells of the interglandular stroma for transcriptome profiling [4]. In addition, a CDw338+ (ABCG2) stem cell population was profiled [7]. These microarray datasets were also deposited in the UESC database [8]. Here, we will illustrate the utility of our UESC database, and a future consortium report will describe in detail the central SCGAP site and its federated search and data analysis tools.

\section{Construction and Content}

The UESC database was based on the Systems Biology Experiment Analysis Management System (SBEAMS) [9], a software and database framework for collecting, storing, and accessing different types of experimental data. SBEAMS combined a relational database management system (RDBMS) back-end, a collection of tools to store, manage, and query experimental information and results, a web front-end for querying the database and providing integrated access to remote data sources, and an interface to other data processing and analysis programs. Since all data from each part of any experiment were organized in a modular schema using similar designs, quality control, analysis, and data integration tasks were greatly simplified. In SBEAMS, each module was made to operate independently but was built upon a core functionality, which included user authentication and auditing, web interface tools, result set management, Gene Ontology integration, centralized BioSequenceSet linking, RDBMS-independence layer, and others. Support for microarrays, proteomics, molecular interactions, macroarrays, gene expression localization, protein functional predictions, and expressed sequence tag (EST) clustering was provided in the current major modules. The SBEAMS module queries were automatically piped to Cytoscape [10] for network visualization and further exploration. Data includes cell type specific information for human and mouse prostate and bladder from immunostaining and microarray. Feedback options and data availability questions are made accessible through contact information listed on the website or through a feedback form.

\section{Populating the database}

The methods of tissue collection, immunostaining and expression data used in this database have been published previously $[3,4,11]$. Briefly, tissue samples consisted of both cancer-enriched and cancer-free samples obtained from over 50 radical prostatectomies or cystectomies under approval by the University of Washington Institutional Review Board following a standard protocol.

\section{Immunohistochemistry data}

The immunohistochemistry data for human and mouse prostate and bladder were annotated and uploaded in the database following the data standard, Minimum Information Specification For In Situ Hybridization and Immuno- 
histochemistry Experiments (MISFISHIE) [11]. The files are systematically named based on the antibody used in staining, the organism, tissue type, tissue block, and magnification. As an example, the file 'CD44 98-395F HP ba 100.jpg' was stained with anti-CD44, was derived from human prostate tissue block 98-395F, is human prostate tissue, and an image of microscopic field of view $b$ within field a was captured at $100 \times$ magnification. Annotation describes the tissue, distribution of reaction product in the tissue, distribution of reaction product in the tissue, localization patterns within histologic cell types, and provides an assessment of the level of expression of the protein for the immunostaining data. Data is available for $200 \mathrm{CD}$ antibodies for human prostate and bladder and 20 CD antibodies for mouse prostate and bladder.

\section{Microarray data}

Affymetrix array analysis of prostate cancer cell lines

The prostate cell transcriptomes of CD26+luminal epithelial, CD $104^{+}$basal epithelial, CD49a ${ }^{+}$stromal fibromuscular, plus $\mathrm{CD} 31^{+}$endothelial, $\mathrm{CDw} 338^{+}$stem, and side population (SP) are all available in UESC $[4,7]$. To date, prostate cancer transcriptomes include those of lineagerelated cancer cell lines LNCaP, C4-2, and CL1, those of PC-3 and DU145, plus that of a CD26+ cancer cell type sorted from a primary tumor of Gleason $3+3$. The bladder transcriptome data includes 1 replicate each of $\mathrm{CD} 13^{+}$ stromal and CD13- stromal cells representing two subdomains of the bladder lamina propria [12]. In the data module, the CEL files are the raw data from the array scan, the RPT files hold the statistical analysis of chip signals, the XML files are MAGE-ML descriptions of the experiments, and the Image files are synthetic JPEG images of the Affymetrix HG-U133_Plus_2 GeneChips.

\section{MPSS analysis of prostate cancer cell lines}

Results from MPSS (Massively Parallel Signature Sequencing) experiments on the $\mathrm{LNCaP}$ and $\mathrm{C} 4-2$ cell lines are also available in the UESC database [13]. Data may be downloaded as an Excel spreadsheet for each cell type, containing the accession number and experimentally detected TPM (transcripts per million) for each analyzed sequence. This data was used to compare MPSS with Affymetrix arrays in their coverage overlap [13].

\section{Searching the database}

\section{$C D$ immunohistochemistry images}

The CD immunohistochemistry images may be viewed or downloaded as ZIP archives. Additionally, a summary of staining for various species and cell types can be downloaded directly as tab-delimited text files. Fig. 1 shows the data summary for CD138 (syndecan-1). Cell type-specific expression is scored by staining intensity, and the uploaded images (at multiple magnifications) of different tissue sections can be opened for examination. Fig. 2 shows CD138 staining at 200× magnification for human bladder and prostate.

\section{Transcriptome data}

The available array datasets (usually after accepted for publication) are listed and can be chosen for interrogation.

(1) Single gene search - in which one can enter Affymetrix Probe Set ID, Gene Name, or Accession Number together with the options of coalescing probesets and replicates. In the Affymetrix HG-U133 arrays often times genes would be represented by multiple probesets, of which not all would give meaningful results. The hybridization signals for all probesets of one gene can be combined if the COALESCE PROBESETS tick box is clicked. The tick box COALESCE REPLICATES averages the signal for each of the biological replicates that make up a sort. The greyscale gradient indicates RMA normalized Affymetrix signal intensity. Signals of 10 or less are represented as white and signals greater than or equal to 10,000 are represented as black. Higher Affymetrix signal (more black) indicates higher levels of gene expression. Fig. 3 shows the analysis output for CD138 (SDC1). Fig. 3A shows the signal intensities scored by the three probesets for SDC1 of all replicates $(n=5)$ of four prostate cell types (CD104+ basal, CD26+luminal, CD $31^{+}$endothelial, CD49a+ stromal) and one replicate each of two bladder cell types (CD13- stromal and $\mathrm{CD}_{13}{ }^{+}$stromal) queried. CD138 expression is detectable in prostate basal cells, and lowered or undetectable in luminal, endothelial and stromal cells. As an illustration of probe variability, the 239256 probeset scored no expression or Absent Call in basal cells (5/5 replicates), and other cell types (5/5 replicates). Fig. 3B shows the analysis summary of CD138 cell-type expression coalesced by replicate, 3C coalesced by probeset, and 3D coalesced by replicate and probeset. This expression data is in accordance with the pattern of prostatic CD138 expression scored by immunohistochemistry (Fig. 1). Included in this summary is the level of CD138 expression in basal urothelial cells.

(2) Multiple gene (batch) search - in which searches can be initiated by using "\%" as a wildcard character (e.g., $\mathrm{CD} \%$ to list all official gene names with the $\mathrm{CD}$ designation) ["_" is a single character wildcard such that, e.g., A_ brings up $\mathrm{AR}$; $\mathrm{A}$ _ brings up $\mathrm{A} 2 \mathrm{M}, \mathrm{ABO}$ to $\mathrm{AXL}, \mathrm{A}$ brings up A1BG to ASB8, etc.]. Fig. 4 shows the query output for gene names with SOX\% (sex determining region $\mathrm{Y}$ box). Other batch search examples would be IL (interleukin), ITG (integrin), TNF (tumor necrosis factor), ADAM (a disintegrin and metalloprotease domain) genes. This query feature is not widely available in many other databases. 


\section{Antibody Summary}

\section{$\underline{\text { CD138 }}$}

Alternate Names: heparan sulfate proteoglycan; syndecan-1

Locus Link: $\quad \underline{6382}$

Genome Coordinates: chr2:20385070-20409385-

Total Assays:

15

\begin{tabular}{|c|c|c|c|c|c|}
\hline Tissue type & Cell type & $\begin{array}{c}\% \\
\text { Intense }\end{array}$ & $\%$ Equivocal & $\%$ None & \# Assays \\
\hline \multirow[t]{8}{*}{ Urinary Bladder } & Cap Cells & 40 & 58 & 3 & 4 \\
\hline & Intermediate Cells & 40 & 54 & 6 & 5 \\
\hline & Basal Epithelial Cells & 40 & 54 & 6 & 5 \\
\hline & Lamina propria - superficial & 0 & 0 & 100 & 6 \\
\hline & Lamina propria - deep & 0 & 0 & 100 & 6 \\
\hline & Submucosa & 1 & 1 & 97 & 7 \\
\hline & Muscularis propria & 0 & 8 & 92 & 6 \\
\hline & Transitional Cell Carcinoma & 0 & 10 & 90 & 1 \\
\hline \multirow[t]{9}{*}{ Prostate } & Atrophic glands & 80 & 20 & 0 & 1 \\
\hline & Hyperplastic glands & 0 & 10 & 90 & 1 \\
\hline & Normal glands & 0 & 10 & 90 & 1 \\
\hline & Basal Epithelial Cells & 60 & 22 & 18 & 3 \\
\hline & Stromal Endothelial Cells & 0 & 0 & 100 & 1 \\
\hline & Stromal Fibromuscular Cells & 5 & 0 & 95 & 1 \\
\hline & Stromal Nerve Sheath Cells & 90 & 5 & 5 & 2 \\
\hline & Stromal Perineural Cells & $\overline{0}$ & $\overline{0}$ & 100 & 1 \\
\hline & Gleason Pattern 3 & 0 & 10 & 90 & 1 \\
\hline
\end{tabular}

\begin{tabular}{|c|c|c|c|c|c|}
\hline Assay Name $+\mathcal{r}$ & Channel Name & & ions & Available Images & \\
\hline CD138 02-034A 5 & CD138 02-034A 5 - chan 1 & + & 8 & $\underline{40 x} 100 x \quad 200 x \quad 200 x \quad 200 x \quad 400 x$ & $\dot{t}$ \\
\hline$\frac{\text { CD138 } 02-047 A}{1}$ & CD138 $02-047 A 1$ - chan 1 & + & & & $\dot{t}$ \\
\hline$\underline{\text { CD138 99-010E } 2}$ & $\underline{\text { CD138 99-010E } 2 \text { - chan } 1}$ & +2 & 10 & & + \\
\hline$\underline{\text { CD138 03-035A1 }}$ & $\underline{\text { CD138 03-035A1 - chan } 1}$ & -1 & 7 & $40 x \quad 100 x \quad 200 x \quad 200 x$ & + \\
\hline$\underline{\mathrm{CD} 13802-054 \mathrm{~A}}$ & $\underline{\text { CD138 02-054A - chan } 1}$ & +2 & & $40 x \underline{100 x} \underline{200 x} \underline{200 x} \underline{200 x} \underline{200 x}$ & + \\
\hline$\underline{\mathrm{CD} 138}$ 03-041B2 & CD138 03-041B2 - chan 1 & -1 & 2 & $\underline{40 x} 100 x \underline{200 x}$ & $\dot{t}$ \\
\hline CD138 03-035A2 & $\underline{\text { CD138 03-035A2 - chan } 1}$ & -1 & 7 & $100 x \quad 200 x \quad 40 x \quad 100 x \quad 200 x \quad 200 x \quad 200 x$ & $\dot{r}$ \\
\hline$\underline{\mathrm{CD} 138 \text { 03-043B1 }}$ & $\underline{\text { CD138 03-043B1 - chan } 1}$ & -1 & 7 & $\underline{100 x} \underline{10 x} \underline{40 x} \underline{100 x} \underline{200 x} \underline{200 x}$ & + \\
\hline$\underline{\text { CD138 02-047A } 1}$ & $\underline{\text { CD138 02-047A } 1 \text { - chan } 1}$ & +2 & & $40 x \quad 100 x \quad 200 x \quad 200 x \quad 200 x \quad 200 x \quad 200 x$ & 4 \\
\hline CD138 03-024A1 & CD138 03-024A1 - chan 1 & + & 6 & $\underline{40 x} \quad \underline{100 x} \underline{200 x} 200 x \quad 400 x \quad 200 x$ & 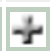 \\
\hline
\end{tabular}

\section{Figure I}

CD immunohistochemistry. Shown is the image data summary for CDI38 (SDCI). The top table provides annotation data including the tissue type, distribution of reaction product in the tissue, localization pattern within histologic cell types and an assessment of the level of protein expression for the immunostaining data. The bottom table provides links to the available images for each annotated sample. Available immunostaining images and additional data can be retrieved by clicking on the links. 

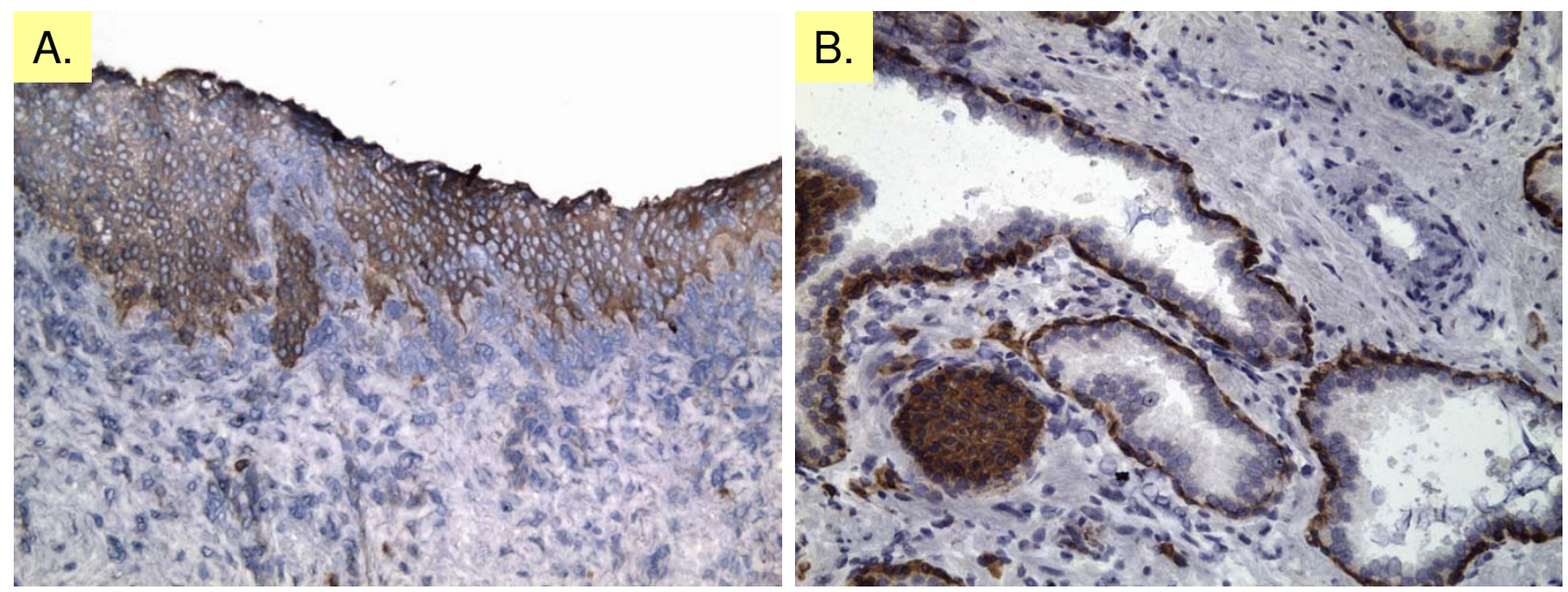

Figure 2

CDI38 immunostaining images. CDI38 (SDCI) immunoreactivity of normal human prostate and bladder (immunoreaction product red-brown; pale blue hematoxylin nuclear counterstain). (A) CDI38 staining of human bladder urothelium, assay name CDI38 03-035AI. (B) CDI 38 staining of human prostate atrophic glands, basal epithelial cells and nerve sheath cells, assay name CDI38 02-007C 5. Original magnification is 200x.

\section{Utility and Discussion}

UESC data types were organized in separate modules to afford a good balance between flexibility and consistency. The management system was designed to allow efficient data access to all levels of users, with both easy web and scriptable, sophisticated interfaces, and to be reusable so that a new project may be built on a previous one (e.g., kidney and bladder cancer data to prostate cancer data).
A.

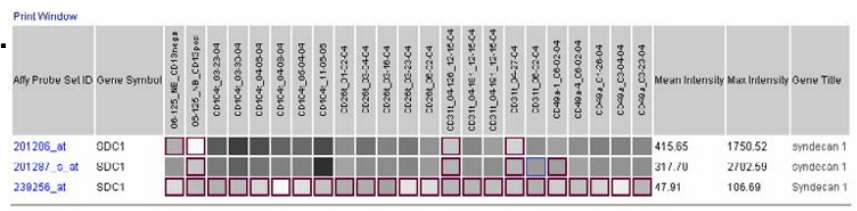

Affy R_CHP Intensity Values Key

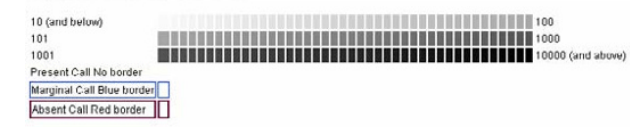

C.

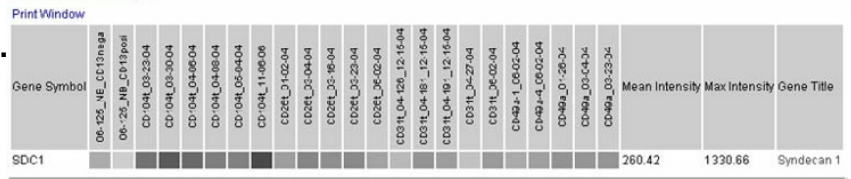

Affy R_CHP Intensity Values Key

10 (and bolow)

101.
B.

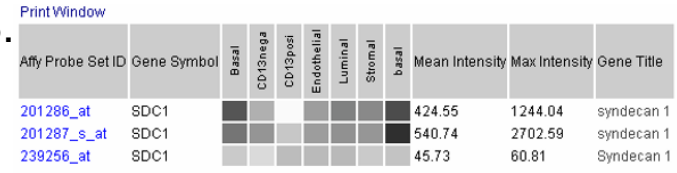

Affy R_CHP Intensity Values Key

10 (and below) 10 (and below)
101 ${ }_{101}^{1001}$

D. Print Window

Figure 3

Single gene search. The expression of CDI38 (SDCI) among the four sorted prostate cell populations (CDI04+ basal epithelial, $C D 26^{+}$luminal epithelial, $C D 3 I^{+}$endothelial and $\mathrm{CD} 3 \mathrm{I}^{+}$stromal fibromuscular) in addition to sorted bladder cell populations ( $\mathrm{CDI} 3^{-}$and $\mathrm{CD} \mathrm{I3+}$ bladder lamina propria) is illustrated. The Affymetrix signal intensity levels are represented by the grey scale. The data can be displayed in full $(\mathbf{A})$, coalesced with respect to biological replicates (B), probesets (C), or both replicate and probeset (D). Present call boxes have no border, Absent calls have a red border, Marginal call is blue (not shown in this example). 
New Search Printable view

\begin{tabular}{|c|c|c|c|c|c|c|c|c|c|c|}
\hline Gene Symbol & 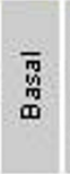 & $\begin{array}{l}\text { m. } \\
\stackrel{D}{0} \\
\stackrel{0}{0} \\
\stackrel{0}{0} \\
0\end{array}$ & $\begin{array}{l}\text { कू } \\
\frac{0}{0} \\
\frac{0}{0}\end{array}$ & 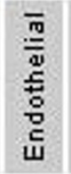 & $\begin{array}{l}\overline{\mathrm{m}} \\
\stackrel{\mathrm{E}}{\mathrm{E}}\end{array}$ & $\begin{array}{l}\overline{\bar{m}} \\
\stackrel{5}{5} \\
\stackrel{5}{0}\end{array}$ & 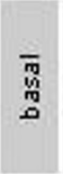 & Mean Intensity & Max Intensity & Gene Title \\
\hline $80 \times 1$ & & & & & & & & 18.39 & 25.38 & SRY (sex determining region $Y$ )-box 1 \\
\hline $80 \times 10$ & & & & & & & & 27.59 & 55.94 & SRY (sex determining region $Y$ )-box 10 \\
\hline sox11 & & & & & & & & 17.35 & 42.05 & SRY (sex determining region Y)-box 11 \\
\hline $\mathrm{s} 0 \times 12$ & & & & & & & & 53.47 & 71.77 & SRY (sex determining region $Y$ )-box 12 \\
\hline $80 \times 13$ & & & & & & & & 77.47 & 109.85 & SRY (sex determining region $Y$ )-box 13 \\
\hline $80 \times 14$ & & & & & & & & 7.52 & 16.89 & SRY (sex determining region $Y$ )-box 14 \\
\hline $80 \times 15$ & & & & & & & & 133.70 & 199.59 & SRY (sex determining region $Y$ )-box 15 \\
\hline $80 \times 17$ & & & & & & & & 441.60 & 1217.69 & SRY (sex determining region $Y$ )-box 17 \\
\hline sox18 & & & & & & & & 42.22 & 98.05 & SRY (sex determining region $Y$ )-box 18 \\
\hline $50 \times 2$ & & & & & & & & 82.42 & 289.90 & SRY (sex determining region $Y$ )-box 2 \\
\hline $50 \times 21$ & & & & & & & & 11.93 & 26.55 & SRY (sex determining region $Y$ )-box 21 \\
\hline SOX2OT & & & & & & & & 6.62 & 12.17 & SOX2 overlapping transcript (non-codi... \\
\hline $50 \times 3$ & & & & & & & & 15.18 & 24.53 & SRY (sex determining region $Y$ )-bo 3 \\
\hline $50 \times 30$ & & & & & & & & 18.58 & 26.00 & SRY (sex determining region $Y$ )-box 30 \\
\hline $50 \times 4$ & & & & & & & & 2340.66 & 2932.89 & SRY (sex determining region $Y$ )-box 4 \\
\hline $80 \times 5$ & & & & & & & & 28.26 & 48.40 & SRY (sex determining region $Y$ )-box 5 \\
\hline $50 \times 6$ & & & & & & & & 40.35 & 45.02 & SRY (sex determining region $Y$ )-box 6 \\
\hline $80 \times 7$ & & & & & & & & 2533.07 & 5104.77 & SRY (sex determining region $Y$ )-box 7 \\
\hline $50 \times 8$ & & & & & & & & 22.92 & 51.99 & SRY (sex determining region $Y$ )-box 8 \\
\hline $80 \times 9$ & & & & & & & & 261.27 & 668.23 & SRY (sex determining region $Y$ )-box 9 .. \\
\hline
\end{tabular}

\section{Affy R_CHP Intensity Values Key}

\section{0 (and below) \\ 101 \\ 1001

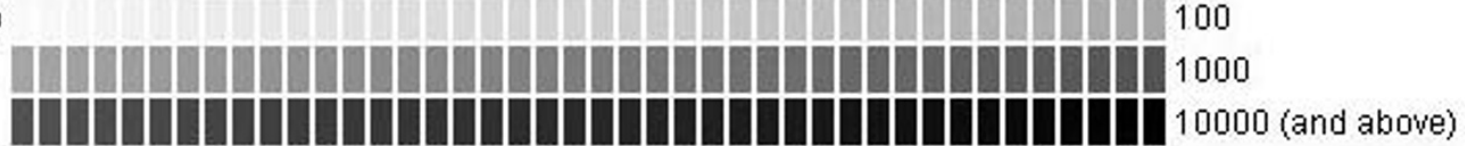

\section{Figure 4}

Multiple gene search. Shown is the query output for gene names containing SOX (sex determining region $Y$ box) among the four sorted prostate cell populations (CD 104+ basal epithelial, CD26+ luminal epithelial, CD3 ${ }^{+}$endothelial and $\mathrm{CD}^{+} \mathrm{I}^{+} \mathrm{stromal}^{+}$ fibromuscular) in addition to sorted bladder cell populations (CDI3-and CDI3+ bladder lamina propria).

The database will therefore continuously expand as more cell type-specific information becomes available. The UESC database will be a valuable tool in the analysis of differential gene expression in prostate cancer genes in cancer progression.

\section{Conclusion}

Strategies for the analysis of the interface between gene expression and protein information involve a variety of computational methods that require the storage and retrieval of large datasets. These databases become perforce an integral component of biomedical research. The UESC database is a unique, web-accessible, searchable compilation of published data concerning the identifica- 
tion and characterization of genes and proteins in specific cell types of the urologic organs where cancer is a major disease. These cell populations retain to a high degree their CD phenotype as determined by immunostaining in intact tissue; concordance between gene expression measured by DNA array and immunohistochemistry was good and will be published separately. These cell type transcriptomes allow us to pursue many studies which are not possible with whole tissue transcriptomes.

\section{Availability and requirements}

The UESC database is freely accessible at http://scgap.sys temsbiology.net/. It has been tested to work with Mozilla Firefox and Internet Explorer.

\section{List of abbreviations used \\ BPH: Benign prostatic hyperplasia}

CD: Cluster designation

EST: Expressed sequence tag

MACS: Magnetic cell sorting

MISFISHIE: Minimum Information Specification For In Situ Hybridization and Immunohistochemistry Experiments

MPSS: Massively Parallel Signature Sequencing

NIDDK: National Institute of Diabetes and Digestive and Kidney Diseases

PEDB: Prostate Expression Database

PGDB: Prostate Gene Database

PIN: Prostatic intraepithelial neoplasia

RDBMS: Relational database management system

RMA: Robust multi-array average

SBEAMS: Systems Biology Experiment Analysis Management System

SCGAP: Stem Cell Genome Anatomy Project

TPM: Transcripts per million

UESC: Urologic Epithelial Stem Cell

\section{Competing interests}

The author(s) declare that they have no competing interests.

\section{Authors' contributions}

LEP drafted the manuscript with input from DSC, EWD and AYL. Database design and programming was performed by EWD, DSC and MK with input from AYL, LEP and LDT. LDT provided immunohistochemistry data annotation and staining summaries. All authors have read and approved the final manuscript.

\section{Acknowledgements}

This work was supported by grant IU0I DK63630 from NIDDK. Additional funding came from grants CA85859, CA98699 and CAIII 244 from $\mathrm{NCl}$. We thank Tracy Sherertz for her contribution to the database construction.

\section{References}

I. Hawkins V, Doll D, Bumgarner R, Smith T, Abajian C, Hood L, Nelson PS: PEDB: the Prostate Expression Database. Nucleic Acids Res 1999, 27(I):204-208

2. Li LC, Zhao H, Shiina H, Kane CJ, Dahiya R: PGDB: a curated and integrated database of genes related to the prostate. Nucleic Acids Res 2003, 3 I ( I ):29|-293.

3. Liu AY, True LD: Characterization of prostate cell types by $C D$ cell surface molecules. Am J Pathol 2002, I60(I):37-43.

4. Oudes AJ, Campbell DS, Sorensen CM, Walashek LS, True LD, Liu AY: Transcriptomes of human prostate cells. BMC Genomics 2006, 7:92.

5. The Stem Cell Genome Anatomy Project [http:// www.scgap.org/]

6. Liu AY, Roudier MP, True LD: Heterogeneity in primary and metastatic prostate cancer as defined by cell surface $C D$ profile. Am J Pathol 2004, 165(5): I543-1556.

7. Pascal LE, Oudes AJ, Petersen TW, Goo YA, Walashek LS, True LD, Liu AY: Molecular and cellular characterization of ABCG2 in the prostate. BMC Urol 2007, 7(I):6.

8. SCGAP Urologic Epithelial Stem Cells Project [http:// scgap.systemsbiology.net/]

9. Marzolf B, Deutsch EW, Moss P, Campbell D, Johnson MH, Galitski $T$ : SBEAMS-Microarray: database software supporting genomic expression analyses for systems biology. BMC Bioinformatics 2006, 7:286.

10. Shannon P, Markiel A, Ozier O, Baliga NS, Wang JT, Ramage D, Amin $\mathrm{N}$, Schwikowski B, Ideker T: Cytoscape: a software environment for integrated models of biomolecular interaction networks. Genome Res 2003, I3(I I):2498-2504.

II. Deutsch EW, Ball CA, Berman J], Bova GS, Brazma A, Bumgarner RE, Campbell D, Causton HC, Christiansen JH, Daian F, Dauga D, Davidson D, Gimenez G, Goo YA, Grimmond S, Henrich T, Gerrmann BG, Johnson MH, Korb M, Mills JC, Oudes AJ, Parkinson HE, Pascal LE, Pollet N, Quackenbush J, Ramialison M, Ringwald M, Salgado D, Sansone SA, Sherlock G, Christian J Stoeckert J, Swedlow J, Taylor RC, Walashek L, Warford A, Wilkinson DG, Zhou Y, Zon LI, Liu AY, True LD: Minimum Information Specification For In Situ Hybridization and Immunohistochemistry Experiments (MISFISHIE). Nature Biotechnology in press.

12. Goo YA, Goodlett DR, Pascal LE, Worthington KD, Vessella RL, True LD, Liu AY: Stromal mesenchyme cell genes of the human prostate and bladder. BMC Urol 2005, 5: 17.

13. Oudes AJ, Roach JC, Walashek LS, Eichner LJ, True LD, Vessella RL, Liu AY: Application of Affymetrix array and Massively Parallel Signature Sequencing for identification of genes involved in prostate cancer progression. BMC Cancer 2005, 5:86.

\section{Pre-publication history}

The pre-publication history for this paper can be accessed here:

http://www.biomedcentral.com/1471-2490/7/19/prepub 